SHORT REPORT

\title{
Arterial hypertension as risk factor for spontaneous cervical artery dissection. A case-control study
}

\author{
A Pezzini, V Caso, C Zanferrari, E Del Zotto, M Paciaroni, C Bertolino, M Grassi, G Agnelli, \\ A Padovani
}

\begin{abstract}
Because of the presumed non-atherosclerotic pathogenesis, the potential link between spontaneous cervical artery dissection (sCAD) and common risk factors for atherosclerosis has never been investigated systematically. Therefore, this prospective, multicentre, case-control study compared the frequency of tobacco use, hypertension, diabetes mellitus, and hypercholesterolaemia among a group of consecutive patients with $\mathrm{SCAD}(\mathrm{n}=153)$, a group of patients with ischaemic stroke, not related to CAD (non-CAD), and a group of controls. As opposed to the other variables, a trend towards a significant association was seen when the prevalence of hypertension was compared among patients with sCAD and controls $(26.8 \%$ v $17.0 \%$; odds ratio (OR) $1.79 ; 95 \%$ confidence interval $(\mathrm{CI}), 0.98$ to 3.27 ; $\mathrm{p}=0.058)$. Hypertension was also significantly associated with the subgroup of patients with $\mathrm{SCAD}$ and cerebral infarction (OR, 1.94; $95 \% \mathrm{Cl}, 1.01$ to $3.70 ; p=0.045)$, particularly when involving the vertebral arteries $(O R, 2.69$; $95 \% \mathrm{Cl}, 1.20$ to $6.04 ; \mathrm{p}=0.017)$. These findings might help define the spectrum of pathogenic conditions predisposing to sCAD and provide information to help investigate the combined effect of such susceptibility factors in future studies.
\end{abstract}

\footnotetext{
C
} ervical artery dissection (CAD) is considered the paradigm of non-atherosclerotic vasculopathies causing cerebral ischaemia. Its putative pathogenic mechanism (an intimal tear that allows circulating blood to enter the arterial wall, splitting its layers, or a primary intramural haematoma), the involvement of arterial segments usually not affected by atherosclerosis, the reported association with heritable connective tissue disorders, and the frequent occurrence in young or middle aged subjects indirectly support this assumption. ${ }^{1}$ Although the aetiology of spontaneous CAD ( $\mathrm{sCAD}$ ) remains poorly understood, an underlying structural defect of the extracellular matrix, resulting from a combination of genetic and environmental factors, is generally suspected. ${ }^{2}$ Conversely, atherosclerosis appears to be rare in patients affected by CAD. For these reasons, the potential link with common risk factors for atherosclerosissuch as tobacco use, hypertension, and diabetes mellitushas never been evaluated systematically. Although evidence from retrospective analyses has indirectly suggested that an association between arterial hypertension and SCAD may exist, $^{34}$ no prospective studies with a control group have specifically explored this hypothesis. Hence, confirmed data on the role of traditional vascular risk factors in the pathogenesis of SCAD are still missing.

Therefore, we undertook the present multicentre, prospective, hospital based, case-control study of a large series of patients affected by SCAD and a series of patients affected by ischaemic stroke not related to a CAD (non-CAD). We investigated the independent contribution of conventional predisposing factors for cerebral ischaemia on the risk of disease and their further influence on specific pathogenic subgroups.

\section{METHODS}

Patients were recruited prospectively from those consecutively admitted to the departments of neurology of the University Hospitals of Brescia, Parma, and Perugia, Italy, between January 1997 and October 2004. The first group consisted of patients with SCAD. All subjects in whom clinical and/or duplex ultrasound findings were consistent with the diagnosis of CAD underwent four vessel conventional angiography and/or magnetic resonance imaging and magnetic resonance angiography of the brain and neck to confirm the diagnosis, as reported previously. ${ }^{5}$ Dissections were classified as spontaneous when occurring spontaneously or in association with a minor trauma. ${ }^{6}$ Patients with a recent history of a major traumatic event were excluded from the analysis. The second group consisted of subjects with ischaemic stroke not related to a CAD (non-CAD) matched to patients with SCAD by age and sex. The group of reference controls consisted of age and sex matched individuals selected from the list of local general practitioners by random digit dialing, after exclusion of subjects with a known history of vascular disease. All control subjects who were asked agreed to participate.

A detailed history was taken from all participating subjects, focusing on vascular risk factors. In particular, demographic data (sex, age), history of arterial hypertension, diabetes mellitus, cigarette smoking, and hypercholesterolaemia before the acute event were considered and assessed according to predefined criteria. ${ }^{5}$ Our study was designed and carried out in observance of the ethical principles established by the local institutional guidelines on clinical investigation. Written, informed consent was provided by all the study participants.

\section{STATISTICAL ANALYSIS}

Differences among the three groups were examined by means of the $\chi^{2}$ test and with the ANOVA $F$ test, when appropriate. A multinomial logistic regression model including age, sex, hypertension, smoking status, diabetes mellitus, and hypercholesterolaemia as explanatory variables was used to examine the differences among the groups of SCAD, nonCAD ischaemic strokes, and controls. Logistic models including hierarchical specific subgroups of patients with SCAD (sCAD, sCAD with cerebral infarct, spontaneous vertebral artery dissection (sVAD) with cerebral infarct) and controls

Abbreviations: $C A D$, cervical artery dissection; $\mathrm{Cl}$, confidence interval; ICAD, internal carotid artery dissection; OR, odds ratio; s, spontaneous; VAD, vertebral artery dissection 
Table 1 Baseline characteristics of the study groups

\begin{tabular}{|c|c|c|c|c|}
\hline Characteristic & sCAD $(n=153)$ & non-CAD IS (n= 153) & Controls $(n=153)$ & p Value \\
\hline Mean (SD) age in years & $45.2(13.1)$ & $44.5(12.9)$ & 44.2 (13.2) & 0.220 \\
\hline Male sex (n) & $86(56.2 \%)$ & $86(56.2 \%)$ & $86(56.2 \%)$ & 0.663 \\
\hline Current smoker (n) & $36(23.5 \%)$ & $57(37.3 \%)$ & $42(27.5 \%)$ & 0.045 \\
\hline Hypertension (n) & $41(26.7 \%)$ & $50(32.7 \%)$ & $26(17.0 \%)$ & 0.041 \\
\hline Diabetes mellitus ( $\mathrm{n}$ ) & $7(4.5 \%)$ & $15(9.8 \%)$ & $5(3.3 \%)$ & 0.209 \\
\hline Hypercholesterolaemia (n) & $41(26.7 \%)$ & $58(37.9 \%)$ & $30(19.6 \%)$ & 0.010 \\
\hline Oral contraceptives* $(n)$ & $15(36.5 \%)$ & $16(31.4 \%)$ & $10(23.3 \%)$ & 0.430 \\
\hline
\end{tabular}

were also used. Data were analysed using the SPSS (version 11.1) program. Results are given as odds ratio (OR) with $95 \%$ confidence intervals (CI). A p value of $<0.05$ on a two sided test was considered significant.

\section{RESULTS}

Each of the three groups comprised 153 subjects. Among patients with SCAD, 136 had a single vessel dissection, involving the internal carotid artery (ICAD) in 84 (54.9\%) patients and the vertebral artery (VAD) in 52 (34.0\%). Multiple vessel dissections were seen in the remaining 17 (11.1\%) patients. One hundred and eight (70.6\%) patients had a complete stroke, whereas a transient ischaemic attack or an oculosympathetic paresis were the only clinical findings in five $(3.2 \%)$ patients and $20(13.0 \%)$ patients, respectively. One hundred and twenty (78.4\%) patients experienced headache and/or neck pain at the time of arterial dissection.

Table 1 summarises the baseline characteristics and history of vascular risk factors in each of the three groups. As expected, patients with non-CAD ischaemic stroke showed a higher frequency of arterial hypertension, diabetes mellitus, and hypercholesterolaemia when compared with both the group of patients with SCAD and the controls. A trend towards a significant association was seen when the prevalence of hypertension was compared between patients with SCAD and controls (26.8\% v 17.0\%; OR, 1.79; 95\% CI, 0.98 to $3.27 ; p=0.058)$. Such an association was more pronounced when the analysis was restricted to patients with sCAD who had computed tomography and/or magnetic resonance imaging confirmed cerebral infarction (31 of 108 $(28.7 \%)$; OR, $1.94 ; 95 \% \mathrm{CI}, 1.01$ to $3.70 ; \mathrm{p}=0.045)$, and to cases of infarct caused by VAD ( 18 of 49 ; OR, 2.61 ; $95 \%$ CI, 1.20 to $6.04 ; \mathrm{p}=0.017)$, as opposed to patients with sCAD without cerebral infarct and patients with ICAD (table 2). Apart from hypertension, none of the other variables under investigation had a significantly higher prevalence in the total group of patients with $\mathrm{SCAD}$, or in specific subgroups, than in the group of controls, and none was significantly higher in the patients with SCAD compared with those with non-CAD ischaemic stroke.

\section{DISCUSSION}

The main findings of our present study are as follows: (1) compared with healthy controls, a history of hypertension was associated with an increased risk of SCAD, but none of the other conventional predisposing factors for stroke seemed to play a role in SCAD pathogenesis; (2) the predisposing effect of hypertension might be dependent on the specific subtype of SCAD, with a more pronounced influence in patients with cerebral infarction, as opposed to those without, and in patients with SVAD as opposed to sICAD.

To date, the potential influence of traditional risk factors for stroke on sCAD occurrence has been largely neglected. The sparse reports comparing the prevalence of these factors between the different subtypes of ischaemic stroke have focused on the most frequent causes of cerebral ischaemia among the elderly, and thus cannot provide information on other defined aetiologies. ${ }^{78}$ To our knowledge, ours is the first study aimed at exploring this aspect in the setting of a case-control design including a specific subgroup of patients with SCAD. The relatively large size of our SCAD sample and the prospective recruitment of patients represent strengths of our analysis. Theoretically, however, a potential inclusion bias cannot be excluded as a result of the hospital based setting. Because the prevalence of risk factors may differ between patients with stroke who are admitted to hospital and those who are not, this might be relevant for the interpretation of our results. In this regard, our findings would require confirmation in a population based stroke incidence study. However, given the relatively low annual incidence of SCAD $(2.5-3 / 100000),{ }^{9}{ }^{10}$ such an approach is not feasible, because the number of subjects required would be prohibitive. Furthermore, the young age of affected individuals and the high frequency of focal signs and symptoms at presentation reduce the possibility that a relevant number of patients with SCAD are not admitted to hospital. A further limitation might be inherent in the noninclusion of proatherogenic factors recently related to SCAD occurrence-such as homocysteine, infection, and migraine $^{11}$-in the final analysis. However, despite the pathogenic implications of these missing data, it seems unlikely that they have altered the results, given the specific purpose of our analysis.

Table 2 Multinomial logistic regression model comparing hierarchical specific subgroups of patients with SCAD with controls

\begin{tabular}{|c|c|c|c|c|c|c|}
\hline \multirow[b]{2}{*}{ Characteristic } & \multicolumn{2}{|c|}{$s C A D(n=153)$} & \multicolumn{2}{|c|}{ sCAD with cerebral infarct $(n=108)$} & \multicolumn{2}{|c|}{ sVAD with cerebral infarct $(n=49)$} \\
\hline & Adjusted OR & $95 \% \mathrm{Cl}$ & Adjusted OR & $95 \% \mathrm{Cl}$ & Adjusted OR & $95 \% \mathrm{Cl}$ \\
\hline Age (years) & 1.00 & 0.98 to 1.02 & 1.00 & 0.98 to 1.02 & 1.01 & 0.98 to 1.03 \\
\hline Male sex & 1.02 & 0.64 to 1.64 & 1.08 & 0.64 to 1.81 & 0.99 & 0.49 to 1.98 \\
\hline Current smoker & 0.77 & 0.45 to 1.31 & 0.87 & 0.49 to 1.54 & 0.71 & 0.32 to 1.60 \\
\hline Hypertension & 1.79 & 0.98 to 3.27 & 1.94 & 1.01 to 3.70 & 2.69 & 1.20 to 6.04 \\
\hline Diabetes mellitus & 1.12 & 0.33 to 3.80 & 1.39 & 0.39 to 4.93 & 1.67 & 0.39 to 7.27 \\
\hline Hypercholesterolaemia & 1.40 & 0.80 to 2.45 & 1.29 & 0.69 to 2.38 & 1.79 & 0.83 to 3.83 \\
\hline
\end{tabular}


There is no simple explanation for the observed association between hypertension and SCAD. In particular, whether dissection is caused by sudden, short lasting, and steep blood pressure changes (a phenomenon that is more likely to occur in hypertensive individuals ${ }^{12}$ ), or whether it is the consequence of the longterm effect of hypertension on the arterial wall (or both), cannot be addressed by our study. An interesting finding from our data is the more pronounced effect of hypertension in patients with sCAD and cerebral infarction as opposed to those without. Why some patients with sCAD develop a cerebral infarct and others do not is unknown at present. One hypothesis is that the dissecting haematoma may extend towards the arterial lumen, causing a stenosis or an occlusion in most of those who develop a cerebral infarct, and towards the adventitia in those who do not. ${ }^{13}$ It might be that the magnitude of the deleterious effect of hypertension on the arterial wall is higher in the internal layers, making the mechanism of SCAD related stenosis or occlusion (and, eventually, cerebral ischaemia) more likely. The high prevalence of hypertension in historical series including mostly patients with cerebral infarction ${ }^{14}$ is fully in line with our results. As to the specific association between hypertension and SVAD related infarcts, it can be speculated that, because of its specific vulnerability to traumatic injury, ${ }^{15}$ the vertebral artery might be more prone to damage by the mechanical effects of hypertension per se. Chronic hypertension might also increase arterial susceptibility to other triggering conditions, such as a minor trauma, a phenomenon that is more likely to occur in the vertebral artery than in the carotid artery. Observational studies ${ }^{15}$ and the sparse familial cases of dissections prompt speculations that sVAD and sICAD might each have specific risk factors, ${ }^{16}$ and further support the assumption that SCAD is a heterogeneous entity in which subtype specific pathogenic mechanisms might operate.

In conclusion, although any hypotheses on the pathogenic mechanisms linking arterial hypertension and SCAD remain speculative at present, we believe our findings help define the spectrum of pathogenic conditions predisposing a cervical artery to dissection and provide information to help investigate the combined effect of such susceptibility factors in future studies.

\footnotetext{
Authors' affiliations

A Pezzini, E Del Zotto, A Padovani, Clinica Neurologica, Università degli Studi di Brescia, P. le Spedali Civili, 1, 25100 Brescia, Italia V Caso, M Paciaroni, G Agnelli, Stroke Unit, Dipartimento di Neuroscienze, Università di Perugia, Via E. dal Pozzo, 06123 Perugia, Italia
}

C Zanferrari, C Bertolino, Istituto di Neurologia, Dipartimento di Neuroscienze, Università di Parma, Strada del Quartiere 4, 43100 Parma, Italia

M Grassi, Dipartimento di Scienze Sanitarie Applicate, Sezione di Statistica Medica ed Epidemiologia, Università degli Studi di Pavia, Pavia, Italia

Competing interests: none declared

Correspondence to: $\operatorname{Dr}$ A Pezzini, Clinica Neurologica, Università degli Studi di Brescia, P. le Spedali Civili, 1, 25100 Brescia, Italia; ale_pezzini@hotmail.com

Received 10 January 2005

Revised version received 23 March 2005

Accepted 4 April 2005

\section{REFERENCES}

1 Schievink WI. Spontaneous cervical artery dissection of the carotid and vertebral arteries. N Engl J Med 2001;344:898-906.

2 Brandt T, Hausser I, Orberk E, et al. Ultrastructural connective tissue abnormalities in patients with spontaneous cervicocerebral artery dissection. Ann Neurol 1998:44:281-5.

3 Konrad C, Muller GA, Langer K, et al. Plasma homocysteine, MTHFR C677T, CBS 844ins68bp, and MTHFDI G1958A polymorphisms in spontaneous cervical artery dissections. J Neurol 2004;251:1242-8.

4 D'Anglejan-Chatillon J, Ribeiro V, Mas JL, et al. Migraine: a risk factor for dissection of cervical arteries. Headache 1989;29:560-1.

5 Pezzini A, Del Zotto E, Archetti S, et al. Plasma homocysteine concentration, C677T MTHFR genotype and 844ins68bp CBS genotype in young adults with spontaneous cervical artery dissection and atherothrombotic stroke. Stroke 2002;33:664-9.

6 Sturzenegger M. Spontaneous internal carotid artery dissection: early diagnosis and management in 44 patients. J Neurol 1995;242:231-8.

7 Bogousslavsky J, Castillo V, Kumral E, et al. Stroke subtypes and hypertension. Primary hemorrhage vs infarction, large vs small-artery disease. Arch Neurol 1996;53:265-9.

8 Schultz UGR, Rothwell PM. Differences in vascular risk factors between etiologic subtypes of ischemic stroke. Importance of population-based studies. Stroke 2003;34:2050-9.

9 Schievink WJ, Mokri B, Whisnant JP. Internal carotid artery dissection in a community: Rochester, Minnesota, 1987-1992. Stroke 1993;24:1678-80.

10 Giroud M, Fayolle H, Andre N, et al. Incidence of internal carotid artery dissection in the community of 'Dijon. I Neurol Neurosurg Psychiatry 1994; 57:1443.

11 Touzé E, Gauvrit JY. Histoire naturelle des dissections des artères cervicales. Revue de la littérature et resultats préliminaires d'une enquete nationale. J Neuroradiol 2002;29:251-6.

12 Mancia G, Parati G, Castiglioni P, et al. Daily life blood pressure changes are steeper in hypertensive than in normotensive subjects. Hypertension 2003;42:277-82

13 Baumgartner RW, Arnold M, Baumgartner I, et al. Carotid dissection with and without ischemic events. Local symptoms and cerebral artery findings. Neurology 2001; $57: 827-32$

14 Mokri B, Sundt TM, Houser OW, et al. Spontaneous dissection of the cervical internal carotid artery. Ann Neurol 1986;19:126-38.

15 Dziewas R, Konrad C, Drager B, et al. Cervical artery dissection. Clinical features, risk factors, therapy and outcome in 126 patients. J Neurol 2003;250:1179-84.

16 Grond-Ginsbach C, Debette S, Pezzini A. Genetic approaches in the study of risk factors for cervical artery dissection. In: Baumgartner RW, Bogousslavsky J, Caso V, et al. Handbook on cerebral artery dissection. Basel: Karger Publishers [In press]. 